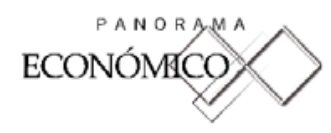

Volumen VII, No. 13

Julio-Diciembre, 2011, pp. 5-22

\title{
CRECIMIENTO ENDÓGENO CON \\ BIENES HETEROGÉNEOS: \\ UN MODELO DE \\ CRECIMIENTO NO BALANCEADO EN LOS DIFERENTES SECTORES DE LA ECONOMÍA
}

\author{
Ignacio Perrotini-Hernández* \\ Francisco Venegas-Martínez** \\ (Recibido: Mayo 2011 / Aprobado: Agosto 2011)
}

\begin{abstract}
Resumen
El presente trabajo desarrolla un modelo de crecimiento endógeno en una economía con consumidores de vida finita que tienen acceso a una canasta de bienes heterogéneos. Las tasas de crecimiento per capita de todos los sectores se caracterizan de manera endógena a través de la tecnología que utilizan las empresas, la tasa subjetiva de descuento, la tasa de reposición del capital, la tasa de crecimiento de la población, el número medio de agentes vivos por unidad de tiempo, la canasta de consumo promedio y la dotación inicial per capita del acervo de capital. Una característica distintiva del modelo propuesto es que éste produce crecimiento no balanceado en los diferentes sectores que conforman la economía.
\end{abstract}

Palabras clave: crecimiento económico, decisiones de los agentes, bienes heterogéneos

* División de Estudios de Posgrado de la Facultad de Economía, Universidad Nacional Autónoma de México. Correo electrónico: <iph@unam.mx>.

** Escuela Superior de Economía, Instituto Politécnico Nacional. Correo electrónico: <fvenegas1111@yahoo.com. $\mathrm{mx}>$. 


\begin{abstract}
This paper develops a model of endogenous growth in an economy with finitely lived consumers who have access to a basket of heterogeneous goods. The per capita growth rates in all sectors are characterized endogenously through the technology used by firms, the subjective discount rate, the rate of replacement of capital, the rate of population growth, the number of live agents per unit of time, the average basket of consumption and the per capita endowment of capital stock. A distinctive feature of the proposed model is that it produces unbalanced growth in different sectors that make up the economy.
\end{abstract}

Keywords: economic growth, agents' decision, heterogeneous goods

Clasificación JEL: F43, D91

\title{
Introducción
}

El desarrollo de la teoría, heterodoxa u ortodoxa, de crecimiento endógeno alcanza su cúspide en la década de los noventa, basta tener en cuenta los trabajos realizados por: Barro (1990), Romer (1994), Barro y Sala-i-Martin (1992), Romer (1990) y Rebelo (1991), entre muchos otros. Así como las investigaciones que incorporan ejercicios de política económica, sobre todo de política fiscal en modelado del crecimiento endógeno, como son: Cazzavillan (1996), Turnovsky (1996), Easterly et al. (1994), Fisher y Turnovsky (1998) y Ludvigson (1996). En la literatura especializada se encuentran también muchos y muy diversos trabajos sobre diferentes aspectos del crecimiento endógeno, por ejemplo: un modelo de crecimiento endógeno no balanceado se encuentra en Eicher y Turnovsky (1999); un modelo con dos sectores es desarrollado por Bond, Wang y Yip (1996); y un modelo de crecimiento endógeno con externalidades se tiene en Benhabib y Jovanovic (1991). Vale la pena destacar que los modelos anteriores fueron desarrollados en ambientes deterministas; en el marco estocástico se pueden mencionar: por ejemplo, las investigaciones de: Reikard (2005), Gokan (2008), Venegas-Martínez (2010) and (2011), Benavie, Grinols y Turnovsky (1996) y Turnovsky (1993), (1998), (1999) y (2000). Por último, se hace notar que existe en la literatura del crecimiento endógeno una visión en donde el gobierno, y no sólo la empresa, puede fomentar la innovación a través 
Crecimiento endógeno con bienes heterogéneos:

un modelo de crecimiento no balanceado en los diferentes sectores de la economía

de programas de actividades de investigación y desarrollo; véanse, por ejemplo: los trabajos de Rivas-Aceves y Venegas-Martínez (2008a), (2008b), (2010a) y (2010b); Venegas-Martínez y Rivas-Aceves (2008) y (2009b), y Rivas-Aceves, Venegas-Martínez y Martínez-Pérez (2009).

Una de las críticas de la heterodoxia hacia la teoría neoclásica, particularmente a la nueva macroeconomía clásica, es que usualmente se postula que los consumidores son idénticos y tienen acceso a un bien genérico de consumo. En este contexto, los consumidores tienen los mismos gustos, dotaciones y vida infinita. El bien genérico se obtiene como un agregado de todos los bienes disponibles en la economía, y la agregación se lleva a cabo a través de la aplicación de los precios relativos (razón entre el precio del bien representativo y el precio del bien que se está agregando) a los otros bienes. Hay también otra crítica sobre la vida infinita de los individuos cuando éstos se conciben como agentes maximizadores de utilidad. Este supuesto se defiende, desde la teoría neoclásica, con el argumento de que realmente no se trata de un individuo que vive para siempre sino de un padre que se preocupa por maximizar la satisfacción de sus hijos, nietos, bisnietos y demás descendientes en un acto de amor filial incondicional e ilimitado, es decir, se preocupa por todas las generaciones futuras, lo cual tiene poco que ver con la realidad, pues nadie está interesado en tomar decisiones de consumo en el presente pensando en los intereses futuros de sus tataranietos. En conclusión, la defensa por parte de la ortodoxia se hace diciendo que se trata de un horizonte de planeación infinito y no de la vida de un individuo. No obstante, la literatura que toma este marco teórico, un bien homogéneo y agentes con vida infinita, es muy abundante y más aún cuando se combinan las expectativas racionales y la racionalidad de los agentes para plantear modelos macroeconómicos de equilibrio general. La discusión sobre estas críticas y muchas otras, así como su defensa, sigue vigente sobre todo desde la apreciación de las visiones institucionalista y evolucionista.

En esta investigación se desarrolla un modelo de crecimiento endógeno para una economía ${ }^{1}$ en donde los individuos tienen acceso a una canasta de bienes heterogéneos, de hecho se supone que existe un continuo de bienes heterogéneos. Asimismo, los agentes que conforman la economía no son idénticos en lo que concierne a que

\footnotetext{
Por simplicidad se toma una economía cerrada dado que la presente investigación se concentra en determinantes del crecimiento diferentes a las exportaciones. Abrir la economía en el modelo propuesto requeriría de modificaciones que complicarían innecesariamente la propuesta.
} 
viven para siempre (o que tiene un horizonte de planeación infinito); aunque sí son iguales en gustos y preferencias, ${ }^{2}$ se supone que los agentes tienen asociada una distribución de probabilidad de que aún estén vivos en un tiempo finito dado. Otra característica distintiva del modelo propuesto es que éste produce crecimiento no balanceado en los diferentes sectores que conforman la economía, es decir, no todos los sectores crecen a la misma tasa.

La gran mayoría de los estudios, teóricos o empíricos, ortodoxos o heterodoxos, sobre crecimiento económico señalan que, entre los determinantes del crecimiento se cuentan la acumulación de capital físico, el capital humano, el avance tecnológico, las innovaciones, las instituciones (reglas del juego) y el "aprendiendo a hacer", entre otros. En este trabajo, las tasas de crecimiento per capita de todos los sectores, en una economía de bienes heterogéneos con agentes de vida finita, se caracterizan, de manera endógena, a través de: 1) la tecnología que utilizan las empresas (mediante un coeficiente tecnológico), 2) la tasa subjetiva de descuento, 3) la tasa de reposición del capital, 4) la tasa de crecimiento de la población, 5) el número medio de agentes vivos por unidad de tiempo, 6) la canasta de consumo promedio y 7) la dotación inicial per capita del acervo de capital. Para ello se combinan la tecnología $A k$ (véanse al respecto, Harrod, 1939 y Rebelo, 1991), con el comportamiento racional de todos los agentes económicos. Es importante destacar que una diferencia entre los modelos típicamente endógenos y el modelo propuesto es que, este último no conduce al crecimiento balanceado.

El presente trabajo está organizado como sigue: en la siguiente sección se establecen los supuestos de la canasta de bienes en cuestión y se obtiene la canasta promedio; en la sección 2 se define la dinámica de crecimiento de la población de la economía bajo estudio; a través de la sección 3 se introduce la distribución exponencial para conducir el tiempo de vida de los agentes; en la sección 4 se proporciona la forma funcional de la utilidad de la canasta promedio de un consumidor con vida finita; en el transcurso de la sección 5 se establece la forma en que la economía asigna recursos a los diferentes sectores que la componen; en la sección 6 se describe el comportamiento de la empresa representativa en la economía; en la sección 7 se especifican las condiciones de primer orden de una solución interior del problema de maximización de utilidad; en la sección 8 se determinan endógenamente las tasas a

2 Un modelo de crecimiento endógeno con agentes heterogéneos en preferencias se encuentra en Ortiz-Arango et al. (2011). 
Crecimiento endógeno con bienes heterogéneos:

un modelo de crecimiento no balanceado en los diferentes sectores de la economía

las que crecen los diferentes sectores de la economía; enseguida se dan las conclusiones, se establecen los alcances y limitaciones del modelo propuesto y se menciona la agenda de investigación futura. Por último, dos apéndices contienen detalles de varios resultados analíticos.

\section{Canasta de bienes heterogéneos y canasta promedio}

Considere una economía cerrada, la cual es poblada con agentes idénticos en preferencias y dotaciones, los cuales son racionales. Al tiempo $t$ los agentes pueden elegir entre diferentes bienes de consumo de una canasta $C_{t}(\gamma), \gamma \in \Gamma$. Es decir, los bienes están indizados por un parámetro $\gamma \in \Gamma$. Si $\Gamma$ es un intervalo cerrado de números reales, entonces hay un continuo de bienes. Se supone que es posible que bienes diferentes tengan asociado un mismo índice. Al tiempo $t$, el bien asociado con el índice $\gamma$ de la canasta está representado por la siguiente relación:

$$
C_{t}(\gamma)=1+C_{t}^{\gamma} \ln \left(C_{t}\right), \quad \gamma \in \Gamma
$$

Si la distribución de los diferentes bienes disponibles en la economía está dada por

$$
f(\gamma)= \begin{cases}\frac{1}{v} & \text { si } \gamma \in \Gamma=[0, v], \\ 0 & \text { si } \gamma \notin[0, v] .\end{cases}
$$

donde $v$ es una constante conocida, en cuyo caso la canasta promedio está representada por $\bar{C}_{t}=\mathrm{E}_{\gamma}\left[C_{t}(\gamma)\right]$, es decir,

$$
\begin{aligned}
\bar{C}_{t} & =\int_{\Gamma} C_{t}(\gamma) f(\gamma) \mathrm{d} \gamma=\frac{1}{v} \int_{0}^{v}\left(1+C_{t}^{\gamma} \ln \left(C_{t}\right)\right) \mathrm{d} \gamma \\
& =\left.\frac{1}{v}\left(\gamma+C_{t}^{\gamma}\right)\right|_{0} ^{v}=\frac{1}{v}\left(v+C_{t}^{v}-1\right)=\frac{C_{t}^{v}}{v}+\left(1-\frac{1}{v}\right) .
\end{aligned}
$$

Bajo el supuesto de que $v$ tiende a 1 , entonces la canasta promedio se puede escribir como 


$$
\bar{C}_{t} \approx \frac{C_{t}^{v}}{v}
$$

En el transcurso de las siguientes secciones estaremos interesados en maximizar la satisfacción de la canasta promedio del agente promedio.

\section{Crecimiento de la población}

En lo que sigue se supone que la población crece a una tasa continua $n$. De esta manera, la dinámica de la población en cada instante satisface:

$$
\frac{\mathrm{d} N_{t}}{N_{t}} \frac{1}{\mathrm{~d} t}=n
$$

Equivalentemente

$$
\dot{N}_{t}=n N_{t}
$$

En otras palabras, la población crece con la siguiente tendencia exponencial

$$
N_{t}=N_{0} e^{n t}
$$

donde $N_{0}$ es el tamaño de la población inicial. Por simplicidad, en lo que sigue, se normalizará la población inicial, esto es, $N_{0}=1$.

\section{Vidas finitas}

A diferencia del planteamiento estándar de individuos con vida infinita, a continuación se define una variable aleatoria $T$ que representa el tiempo de vida del agente. Se supone que la probabilidad de que el agente esté vivo al tiempo $t$ satisface:

$$
P\{T>t\}=e^{-\beta t}
$$

donde $\beta$ es el número medio de agentes vivos, al tiempo $t$, por unidad de tiempo. Es decir $T$ sigue una función de densidad de la forma: 


$$
f_{T}(t)=\left\{\begin{array}{lll}
\beta e^{-\beta t} & \text { si } & t>0, \\
0 & \text { si } & t \leq 0 .
\end{array}\right.
$$

De tal forma que

$$
f_{T}(t)=\frac{\mathrm{d}}{\mathrm{d} t} F_{T}(t)=\frac{\mathrm{d}}{\mathrm{d} t} P\{T \leq t\}=\frac{\mathrm{d}}{\mathrm{d} t}(1-P\{T>t\})=\frac{\mathrm{d}}{\mathrm{d} t}\left(1-e^{-\beta t}\right)=\beta e^{-\beta t} .
$$

En otras palabras, la función de densidad exponencial de parámetro $\beta$ conduce el tiempo de vida de los agentes.

\section{Utilidad de la canasta promedio del agente con vida finita}

Se supone ahora que existe un planeador central que desea maximizar la satisfacción del agente promedio cuando este está vivo al tiempo $t$. Específicamente, se supone que el planeador central desea maximizar

$$
U=\int_{t=0}^{\infty} u\left(\bar{C}_{t} / N_{t}\right) e^{-(\rho-n) t} P\{T>t\} \mathrm{d} t,
$$

donde $u\left(\bar{C}_{t} / N_{t}\right)$ es la función de utilidad sobre la canasta promedio de consumo per capita $\bar{c}_{t}=\bar{C}_{t} / N_{t}$, en la que $N_{t}$ es el consumo agregado y $\rho$ es la tasa subjetiva de descuento (o tasa intertemporal de descuento), la cual es la misma para todos los consumidores. Observe que la utilidad también se descuenta considerando las generaciones futuras a través de la tasa de crecimiento de la población $n$. Se supone también que la función de utilidad toma específicamente la siguiente forma funcional:

$$
u\left(\bar{c}_{t}\right)=-e^{-\bar{c}_{t}^{1 / v}}
$$

equivalentemente

$$
u\left(\bar{c}_{t}\right)=-e^{-\theta c_{t}}, \quad \theta>0
$$

donde $\theta=v^{-1 / v}$. Observe que el parámetro $\theta$ representa la canasta promedio. 


\section{Asignación de recursos}

Ahora bien, se supone que la asignación de recursos está dada por la identidad de la renta nacional (para una economía cerrada) y no por un sistema de precios. De esta manera,

$$
Y_{t}=C_{t}+I_{t}+G_{t}
$$

donde $Y_{t}$ es el producto interno bruto, $I_{t}$ es la inversión neta (los bienes nuevos que se destinan a la producción) y $G_{t}$ es el gasto de gobierno. La inversión neta está dada por

$$
I_{t}=\dot{K}_{t}+\delta K_{t}
$$

donde $\dot{K}_{t}$ es la inversión bruta y $\delta$ es la tasa de depreciación del capital. Después de sustituir la ecuación (7) en la expresión (6), se tiene en términos per capita que:

$$
\frac{Y_{t}}{N_{t}}=\frac{C_{t}}{N_{t}}+\frac{\dot{K}_{t}}{N_{t}}-\delta \frac{K_{t}}{N_{t}}+\frac{G_{t}}{N_{t}}
$$

Sea $k_{t}=K_{t} / N_{t}$ el capital per capita. De hecho, de ahora en adelante, todas las variables expresadas en letras minúsculas se referirán a cantidades per capita (a menos que se especifique lo contrario). Observe, primero, que

$$
\dot{k}_{t}=\frac{\mathrm{d}}{\mathrm{d} t}\left(\frac{K_{t}}{N_{t}}\right)=\frac{N_{t} \dot{K}_{t}-K_{t} \dot{N}_{t}}{N_{t}^{2}}=\frac{\dot{K}_{t}}{N_{t}}-\frac{K_{t}}{N_{t}} \frac{\dot{N}_{t}}{N_{t}}=\frac{\dot{K}_{t}}{N_{t}}-n k_{t}
$$

de donde

$$
\frac{\dot{K}_{t}}{N_{t}}=\dot{k}_{t}+n k_{t}
$$

Al sustituir la ecuación anterior en (8), se tiene que la producción per cápita satisface:

$$
y_{t}=c_{t}+\dot{k}_{t}+\delta k_{t}+n k_{t}+g_{t}
$$


Crecimiento endógeno con bienes heterogéneos:

un modelo de crecimiento no balanceado en los diferentes sectores de la economía

Por simplicidad se supone que la economía es una autarquía, en cuyo caso $g_{t}=0$, es decir

$$
\dot{k}_{t}=y_{t}-(\delta+n) k_{t}-c_{t}
$$

Observe que la producción se destina a tres fines: el consumo, la inversión junto con la depreciación y la dotación de capital inicial a las nuevas generaciones.

\section{Comportamiento de las empresas}

En lo que sigue se supone que la producción se lleva a cabo por una empresa representativa que utiliza la tecnología

$$
y_{t}=f\left(k_{t}\right)=A k_{t}
$$

Es decir, el producto marginal del capital es constante e igual a $A$. En lo que sigue, se supondrá que en todo momento $y_{t}=f\left(k_{t}\right)$. El valor presente, $V P$, de la empresa representativa está dado por

$$
V P=\int_{t=0}^{\infty}\left(A k_{t}-r k_{t}\right) e^{-r t} \mathrm{~d} t
$$

donde $r$ es el rendimiento que paga el capital. La condición de primer orden del problema de maximización del $V P$ conduce a:

$$
A=r
$$

Así, el producto marginal del capital satisface que $A=r$.

\section{Condiciones de primer orden del problema de maximización de utilidad}

En esta sección se plantea el problema que resuelve el planeador central, interesado en maximizar la satisfacción del individuo promedio. Así pues, con base en las ecuaciones (1)-(5) y (12), el problema de decisión del planeador central está dado por: 


$$
\begin{gathered}
\text { Maximizar } U=\int_{t=0}^{\infty}-e^{-\theta c_{t}} e^{-(\rho+\beta-n) t} \mathrm{~d} t, \\
\text { sujeto a: } \dot{k}_{t t}=(A-\delta-n) k_{t}-c_{t}, \quad k_{0} \text { dado. }
\end{gathered}
$$

donde $k_{0}$ es la dotación inicial del consumidor. En este caso, el Hamiltoniano del problema anterior de control óptimo es

$$
H=-e^{-\theta c_{t}}+\lambda_{t}\left[(A-\delta-n) k_{t}-c_{t}\right]
$$

donde $\lambda_{t}$ es el multiplicador (precio sombra) asociado a la restricción. Las condiciones de primer orden para una solución interior son:

$$
\frac{\partial H}{\partial c_{t}}=0, \quad-\frac{\partial H}{\partial k_{t}}=\dot{\lambda}_{t}-\lambda_{t}(\rho+\beta-n) \quad \text { y } \quad \frac{\partial H}{\partial \lambda_{t}}=\dot{k}_{t} .
$$

La primera ecuación de (17) (llamada condición del control óptimo) conduce a

$$
\theta e^{-\theta c_{t}}-\lambda_{t}=0
$$

Asimismo, la segunda condición en (17) (llamada la ecuación adjunta) conlleva a

$$
-\lambda_{t}(A-\delta-n)=\dot{\lambda}_{t}-(\rho+\beta-n) \lambda_{t}
$$

Equivalentemente,

$$
\dot{\lambda}_{t}=(\rho+\delta+\beta-A) \lambda_{t}
$$

Observe que la variable de coestado $\lambda_{t}$ es independiente de la tasa de crecimiento de la población. La ecuación diferencial ordinaria de primer orden homogénea, dada en (19), tiene solución, con condición inicial $\lambda_{0}$ al tiempo $t=0$, dada por:

$$
\lambda_{t}=\lambda_{0} e^{(\rho+\delta+\beta-A) t}
$$

Observe que (18) conduce a 
Crecimiento endógeno con bienes heterogéneos:

un modelo de crecimiento no balanceado en los diferentes sectores de la economía

$$
c_{t}=\frac{\ln (\theta)-\ln \left(\lambda_{t}\right)}{\theta}
$$

y al sustituir (20) en la ecuación anterior se tiene que

$$
c_{t}=\frac{\ln (\theta)-\ln \left(\lambda_{0} e^{(\rho+\delta+\beta-A) t}\right)}{\theta}
$$

es decir,

$$
\begin{aligned}
c_{t}= & \frac{\ln (\theta)-\ln \left(\lambda_{0}\right)-(\rho+\delta+\beta-A) t}{\theta} \\
& =\frac{\ln (\theta)-\ln \left(\lambda_{0}\right)}{\theta}-\frac{(\rho+\delta+\beta-A) t}{\theta} .
\end{aligned}
$$

Ahora bien, si se supone que la siguiente condición de transversalidad ${ }^{3}$ se cumple (véase el Apéndice A)

$$
\lim _{t \rightarrow \infty} k_{t} e^{-(A-\delta-n) t}=0
$$

entonces

$$
\int_{0}^{\infty} c_{t} e^{-(A-\delta-n) t} \mathrm{~d} t=k_{0}
$$

en cuyo caso se sigue que

$$
k_{0}=\frac{1}{\theta}\left[\frac{\ln (\theta)-\ln \left(\lambda_{0}\right)}{A-\delta-n}-\frac{(\rho+\delta+\beta-A)}{(A-\delta-n)^{2}}\right] .
$$

En consecuencia, la trayectoria óptima de consumo satisface

$$
\begin{aligned}
c_{t}= & \frac{\ln (\theta)-\ln \left(\lambda_{0}\right)-(\rho+\delta+\beta-A) t}{\theta} \\
& =(A-\delta-n) k_{0}-\frac{A-\rho-\delta-\beta}{\theta(A-\delta-n)}+\frac{A-\rho-\delta-\beta}{\theta} t .
\end{aligned}
$$

3 Esta condición establece que el individuo no puede hacer pirámides para endeudarse indefinidamente pagando intereses contratando más y más deuda. 
Es decir, el consumo es lineal en el tiempo. Con el fin de mantener el consumo positivo se supone que

$$
(A-\delta-n) k_{0}>\frac{A-\rho-\delta-\beta}{\theta(A-\delta-n)}>0 .
$$

Por otro lado, a partir de la restricción presupuestal dada en (11), se tiene la ecuación que determina la dinámica del capital,

$$
\begin{aligned}
\dot{k}_{t} & =(A-\delta-n) k_{t}-c_{t} \\
& =(A-\delta-n) k_{t}-\left((A-\delta-n) k_{0}+\frac{\rho+\delta+\beta-A}{\theta(A-\delta-n)}-\frac{(\rho+\delta+\beta-A) t}{\theta}\right) .
\end{aligned}
$$

La solución de esta ecuación diferencial ordinaria no homogénea está dada por (ver el Apéndice B)

$$
k_{t}=k_{0}+\frac{(A-\rho-\delta-\beta)}{\theta(A-\delta-n)} t>0
$$

\section{Tasas de crecimiento de los sectores}

Si se denota la tasa de crecimiento de un variable $x_{t}$ mediante $\xi_{x}=\dot{x}_{t} / x_{t}$, entonces, a partir de la ecuación (24), se sigue que la tasa de crecimiento del consumo satisface:

$$
\xi_{c}=\frac{A-\rho-\delta-\beta}{\theta c_{t}}>0 .
$$

Del mismo modo, con base en la expresión (25), se tiene que la tasa de crecimiento del capital está dada por:

$$
\xi_{k}=\frac{(A-\rho-\delta-\beta)}{\theta(A-\delta-n) k_{t}}>0 .
$$

Ahora bien en virtud de la ecuación (12) se obtiene, finalmente, que

$$
\xi_{y}=\frac{(A-\rho-\delta-\beta)}{\theta(A-\delta-n) A k_{t}}>0 .
$$


Observe que la tasa de crecimiento per capita del producto depende de la tecnología que utilizan las empresas (mediante un coeficiente tecnológico), la tasa subjetiva de descuento, la tasa de reposición del capital, la tasa de crecimiento de la población, el número medio de agentes vivos por unidad de tiempo, la canasta promedio y la dotación inicial per capita del acervo de capital. Por último, note que el modelo desarrollado no conduce al crecimiento balanceado, es decir, todos los sectores crecen a diferentes tasas.

\section{Conclusiones}

Este trabajo ha desarrollado un modelo de crecimiento endógeno en una economía con bienes heterogéneos. En el modelo propuesto se maximiza la satisfacción de la canasta promedio de un agente con vida finita. Las tasas de crecimiento per capita de todos los sectores en la economía se caracterizaron, de manera endógena, a través de la tecnología, la tasa subjetiva de descuento, la tasa de reposición del capital, la tasa de crecimiento de la población, el número medio de agentes vivos por unidad de tiempo, la canasta promedio y la dotación inicial per capita del acervo de capital.

En el modelo propuesto se combinaron la tecnología $A k$ con el comportamiento racional de todos los agentes económicos. Una característica distintiva del modelo es que no conduce al crecimiento balanceado, es decir, los sectores crecen a diferentes tasas. Con el fin de obtener soluciones analíticas de las tasas de crecimiento se supuso una forma funcional específica para el índice de satisfacción, a saber, la exponencial negativa. Una limitación del modelo es que los resultados dependen de la función de utilidad escogida; es de esperarse, por ejemplo, que con utilidad logarítmica los resultados sean diferentes.

Por supuesto, el modelo propuesto se desarrolló en un ambiente determinista y es necesario extenderlo a ambientes con riesgo e incertidumbre. Por ejemplo, se podría incluir un activo riesgoso (un título de capital de una emisora representativa que cotiza en bolsa) cuyo precio puede ser conducido por el movimiento geométrico browniano; incluso se podría suponer una volatilidad estocástica con reversión a la media. Asimismo, se podrían incluir saltos en el precio de la acción mediante un proceso homogéneo de Poisson. Por último, se podría incluir incertidumbre en la política fiscal futura a través de un proceso Markoviano de difusión que conduzca la tasa impositiva sobre la riqueza real del individuo. 


\section{Bibliografía}

Barro, R. (1990), "Government Expending in a Simple Model of Endogenous Growth", Journal of Political Economy, vol. 98, núm. 5, pp. S103-S125.

Barro, R. y X. Sala-i-Martin (1992), "Public Finance in Models of Economic Growth", Review of Economic Studies, vol. 59, núm. 4, pp. 654-661.

Benavie, A., E. Grinols y S. Turnovsky (1996), “Adjustment Costs and Investment in a Stochastic Endogenous Growth Model” Journal of Monetary Economics, vol. 38, núm. 1, pp. 77-100.

Benhabib, J. y B. Jovanovic (1991), "Externalities and Growth Accounting”, American Economic Review, vol. 81, núm. 1, pp. 82-113.

Bond W., P. Wang y K. Yip (1996), "A General Two-Sector Model of Endogenous Growth with Human and Physical Capital: Balanced Growth and Transitional Dynamics", Journal of Economic Theory, vol. 68, núm. 1, pp. 149-173.

Cazzavillan, G. (1996), "Public Spending, Endogenous Growth and Endogenous Fluctuations", Journal of Economic Theory, vol. 71, núm. 2, pp. 394-415.

Easterly, W., R. King, R. Levine y S. Rebelo (1994), Policy, Technology Adoption and Growth, Economic Growth and the Structure of Long-Term Development, Proceedings of the IEA Conference. Varenna Italy, pp. 75-89.

Eicher, T. y S. Turnovsky (1999), "Non-Scale Models of Economic Growth", Economic Journal, vol. 109, núm. 457, pp. 394-415.

Fisher, W. y S. Turnovsky (1998), "Public Investment, Congestion, and Private Capital Accumulation", The Economic Journal, vol. 108, núm. 447, pp. 399-413.

Gokan, Y. (2008), "Infrastructure, Alternative Government Finance and Stochastic Endogenous Growth", Journal of economic Dynamics and Control, vol. 32, núm. 2, pp. 321-347.

Harrod, R. (1939), “An Essay in Dynamic Theory”, The Economic Journal, vol. 49, núm. 193, pp. 14-33.

Ludvigson, S. (1996), "The Macroeconomic Effects of Government Debt in a Stochastic Growth Model”, Journal of Monetary Economics, vol. 38, núm. 1, pp. 25-45.

Ortiz-Arango, F., F. Venegas-Martínez y F. López-Herrera (2011), “Crecimiento endógeno no balanceado con agentes heterogéneos", capítulo del libro Crecimiento, desarrollo y crisis financiera: Una visión crítica de la Economía, Coordinadores F. Ortiz-Arango y A. Ortiz-Ramírez. Universidad Panamericana. Por aparecer. 
Crecimiento endógeno con bienes heterogéneos:

un modelo de crecimiento no balanceado en los diferentes sectores de la economía

Rebelo, S. (1991), "Long-Run Policy Analysis and Growth", Journal of Political Economy, vol. 99, núm. 3, pp. 500-521.

Reikard, G. (2005), "Endogenous Technical Advance and the Stochastic Trend in Output: A Neoclassical Approach", Research Policy, vol. 34, núm. 10, pp. 1476-1490.

Rivas-Aceves, S. y F. Venegas-Martínez (2008a), "Participación del gobierno en el desarrollo tecnológico en un modelo de crecimiento endógeno de una economía monetaria", Problemas del Desarrollo, Revista Latinoamericana de Economía, vol. 39, núm. 152, pp. 47-68.

Rivas-Aceves, S. y F. Venegas-Martínez (2008b), "Estabilización de precios y expectativas de devaluación: tasas de interés estocásticas", Análisis Económico, vol. 23. núm. 53, pp. 99-110.

Rivas-Aceves, S. y F. Venegas-Martínez (2010a), “Gobierno como promotor del cambio tecnológico: un modelo de crecimiento endógeno con trabajo, dinero y deuda". Economía Mexicana, Nueva Época, vol. 19, núm. 1, pp. 91-117.

Rivas-Aceves, S. y F. Venegas-Martínez (2010b), "Cambio tecnológico financiado por el gobierno: un modelo de crecimiento endógeno", Región y Sociedad, vol. 22, núm. 49, pp. 91-116.

Rivas-Aceves, S., F. Venegas-Martínez y J. F. Martínez Pérez (2009), “Gobierno, cambio tecnológico y difusión tecnológica”, Equilibrio Económico, Año X, vol. 5 núm. 2, pp. 189-216.

Romer, P. (1990), "Endogenous Technological Change", The Journal of Political Economy, vol. 98, núm. 5, Part 2, pp. S71-S102.

Romer, P. (1994), “The Origins of Endogenous Growth", The Journal of Economic Perspectives, vol. 8, núm.1, pp. 3-22.

Turnovsky, S. (1993), "Macroeconomic Policies, Growth, and Welfare in a Stochastic Economy”, International Economic Review, vol. 34, núm. 4, pp. 953-981.

Turnovsky, S. (1996), "Optimal Tax, Debt, and Expenditure Policies in a Growing Economy”. Journal of Public Economics, vol. 60, núm. 1, pp. 21-44.

Turnovsky, S. (1998), "Productive Government Expenditure in a Stochastically Growing Economy”. Macroeconomic Dynamics, vol. 3, núm. 4, pp. 544570.

Turnovsky, S. (1999), "On the Role of Government in a Stochastically Growing Open Economy", Journal of Economic Dynamics and Control, vol. 23, núm. 5-6, pp. 873-908. 
Turnovsky, S. (2000), "Government Policy in a Stochastic Growth Model with Elastic Labor Suppl”, Journal of Public Economic Theory, vol. 2, núm. 4, pp. 389433.

Venegas-Martínez, F. (2010), "Fiscal Policy in a Stochastic Model of Endogenous Growth: the Mexican Case", Indian Development Review: An International Journal of Development Economics, vol. 8, núm.1-2, pp. 139-157.

Venegas-Martínez, F. (2011), How Risk Factors Affect Growth in Mexico: A FreeMarket Liberalism Approach, Chapter 10: "Market Liberalism, Growth and Economic Development in Latin America”, Eds. G. Ángeles-Castro, I. Perrotini-Hernández y H. Ríos-Bolivar, Routledge, pp. 220-232.

Venegas-Martínez, F. y S. Rivas-Aceves (2009b), "El gobierno como promotor del cambio tecnológico: un modelo de crecimiento con aprendizaje", Eseconomía, Revista de Estudios Económicos, Tecnológicos y Sociales, núm. 22, pp. 7-29.

Venegas-Martínez, F. y S. Rivas-Aceves (2008), "Impulso tecnológico gubernamental en la agroindustria: un modelo de crecimiento endógeno", Portes, Revista Mexicana de Estudios de la Cuenca del Pacífico, vol. 2, núm. 3, pp. 203-234. 


\section{Apéndice A}

En el presente apéndice se presentan los detalles matemáticos de la ecuación (23).

$$
\begin{aligned}
k_{0}-\lim _{t \rightarrow \infty} k_{t} e^{-(A-\delta-n) t} & =\int_{0}^{\infty}\left(\frac{\ln (\theta)-\ln \left(\lambda_{0}\right)-(\rho+\delta+\beta-A) t}{\theta}\right) e^{-(A-\delta-n) t} \mathrm{~d} t \\
& =\left(\frac{\ln (\theta)-\ln \left(\lambda_{0}\right)}{\theta}\right) \int_{0}^{\infty} e^{-(A-\delta-n) t} \mathrm{~d} t-\frac{(\rho+\delta+\beta-A)}{\theta} \int_{0}^{\infty} t e^{-(A-\delta-n) t} \mathrm{~d} t \\
& =\left(\frac{\ln (\theta)-\ln \left(\lambda_{0}\right)}{\theta(A-\delta-n)}\right)-\frac{(\rho+\delta+\beta-A)}{\theta(A-\delta-n)^{2}}
\end{aligned}
$$

Este resultado coincide con la expresión (23).

\section{Apéndice B}

En este apéndice se obtiene, detalladamente, la ecuación (29). Note primero que

$$
\begin{aligned}
\dot{k}_{t}=(A-\delta-n) k_{t}-c_{t}= & (A-\delta-n) k_{t} \\
& -\left((A-\delta-n) k_{0}+\frac{\rho+\delta+\beta-A}{\theta(A-\delta-n)}-\frac{(\rho+\delta+\beta-A) t}{\theta}\right) .
\end{aligned}
$$

Se definen las siguientes constantes a fin de hacer la notación más simple. Sean

$$
\begin{gathered}
A_{0}=A-\delta-n, \\
B_{0}=(A-\delta-n) k_{0}+\frac{\rho+\delta+\beta-A}{\theta(A-\delta-n)}=A_{0} k_{0}+\frac{\rho+\delta+\beta-A}{\theta A_{0}}
\end{gathered}
$$

y

$$
D_{0}=\frac{\rho+\delta+\beta-A}{\theta}
$$

Note ahora que 


$$
\frac{B_{0}}{A_{0}}=k_{0}+\frac{\rho+\delta+\beta-A}{\theta}
$$

$\mathrm{y}$

$$
\frac{B_{0}}{A_{0}}=(A-\delta-n) k_{0}+\frac{\rho+\delta+\beta-A}{\theta(A-\delta-n)}=k_{0}+\frac{D_{0}}{A_{0}^{2}}
$$

así

$$
\frac{D_{0}}{A_{0}^{2}}=\frac{B_{0}}{A_{0}}-k_{0}
$$

Y de esta manera,

$$
\dot{k}_{t}=A_{0} k_{t}-B_{0}+D_{0} t .
$$

La solución de la ecuación diferencial ordinaria no homogénea de primer orden está dada por

$$
\begin{aligned}
k_{t} & =k_{0} e^{A_{0} t}-B_{0} e^{A_{0} t} \int_{0}^{t} e^{-A_{0} s} \mathrm{~d} s+D_{0} e^{A_{0} t} \int_{0}^{t} s e^{-A_{0} s} \mathrm{~d} s \\
& =k_{0} e^{A_{0} t}-\frac{B_{0}}{A_{0}} e^{A_{0} t}\left(1-e^{-A_{0} t}\right)+D_{0} e^{A_{0} t} \int_{0}^{t} s e^{-A_{0} s} \mathrm{~d} s \\
& =k_{0} e^{A_{0} t}-\frac{B_{0}}{A_{0}} e^{A_{0} t}\left(1-e^{-A_{0} t}\right)+D_{0} e^{A_{0} t}\left(-\left.\frac{1}{A_{0}} s e^{-A_{0} s}\right|_{0} ^{t}+\frac{1}{A_{0}^{2}} \int_{0}^{t} A_{0} e^{-A_{0} s} \mathrm{~d} s\right) \\
& =k_{0} e^{A_{0} t}-\frac{B_{0}}{A_{0}}\left(e^{A_{0} t}-1\right)+\left(-\frac{D_{0}}{A_{0}} t+\frac{D_{0}}{A_{0}^{2}}\left(e^{A_{0} t}-1\right)\right) \\
& =k_{0} e^{A_{0} t}-\frac{B_{0}}{A_{0}}\left(e^{A_{0} t}-1\right)+\left(-\frac{D_{0}}{A_{0}} t+\left(\frac{B_{0}}{A_{0}}-k_{0}\right)\left(e^{A_{0} t}-1\right)\right) \\
& =k_{0}-\frac{D_{0}}{A_{0}} t .
\end{aligned}
$$

lo cual coincide con la ecuación (25). 